\title{
Correction: Congenital adrenal hyperplasia due to 11-beta-hydroxylase deficiency: functional consequences of four CYP11B1 mutations
}

Soara Menabò $\cdot$ Seher Polat $\cdot$ Lilia Baldazzi - Alexandra E. Kulle · Paul-Martin Holterhus · Joachim Grötzinger • Flaminia Fanelli • Antonio Balsamo · Felix G. Riepe

Published online: 18 February 2020

(c) European Society of Human Genetics 2020

Correction to: European Journal of Human Genetics https://doi.org/10.1038/ejhg.2013.197
In the originally published article, there are two cases where the pathogenic variant p.(Arg332Gln) was written as p.(Arg332Trp). The two cases of p.(Arg332Trp) should therefore be p.(Arg332Gln) in the original manuscript. 